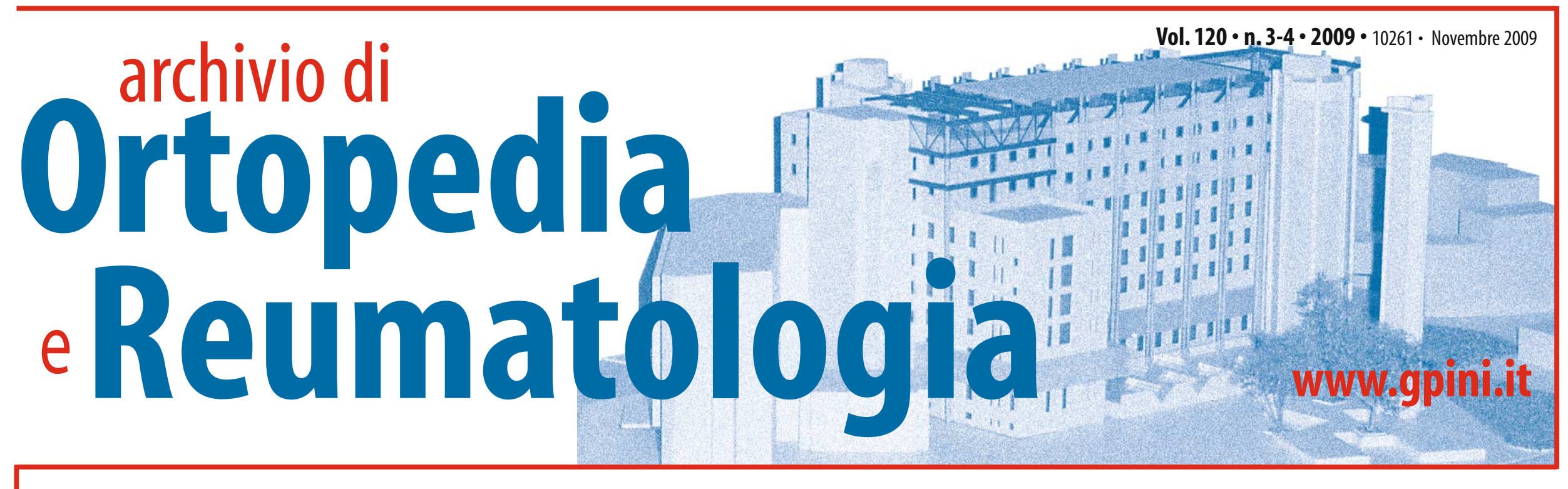

\title{
Biotecnologie applicate alla patologia articolare del ginocchio
}

\section{Editoriale}

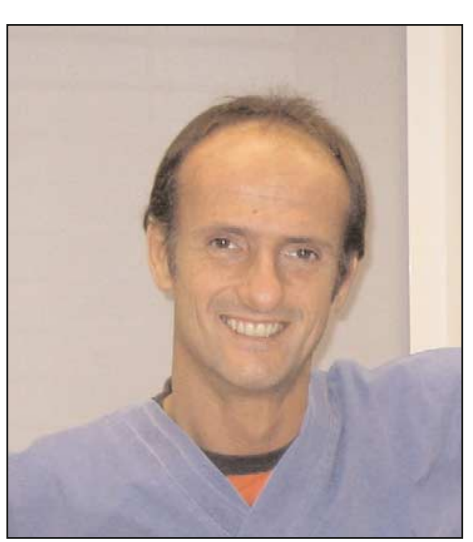

M. Berruto

embra passato un secolo da quel numero del "New England Journal of Medicine" del 1994 in cui Brittberg, Peterson e coll. (N Engl J Med 1994; 331:889-895) pubblicavano i primi risultati di quella che allora sembrava una tecnica rivoluzionaria: il trapianto autologo di condrociti. Quelle immagini di ginocchia "aperte" con "patch" di periostio applicato e suturato alla cartilagine circostante a chiudere una tasca dove venivano inserite cellule cartilaginee in sospensione liquida precedentemente prelevate, sembrano appartenere alla preistoria dell'ortopedia. Eppure sono passati appena 15 anni. E in questi 15 anni quella tecnica un po' "primitiva" ha dimostrato una sua almeno parziale validità, ma soprattutto, come accade a ogni pioniere, ha aperto una nuova via, stimolando la ricerca di soluzioni più avanzate, meno invasive, più efficaci. I "cavalieri svedesi", come in un film ambientato nel vecchio West, hanno spostato il confine dell'ortopedia tradizionale più in là, aprendo gli orizzonti e gli sguardi su quella nuova val- le che oggi definiamo con il termine di "biotech". Una valle vergine, tutta da scoprire e da esplorare, ricca di promesse e di suggestioni, probabilmente anche di trappole e di illusioni. Ma è indubbio che oggi il "biotech" rappresenti una concreta speranza, una miniera in cui le menti speculative, la ricerca e le energie applicative fondono le proprie energie con l'intento di trovare finalmente il loro Eldorado: l'agognata e sognata rigenerazione tissutale.

E il ginocchio, in ambito articolare, un po' come avvenne ai tempi dell'altra grande rivoluzione, quella della chirurgia artroscopica, oggi come allora, rappresenta il terreno ideale su cui far convergere le idee, l'impegno e le energie.

Oggi parlare di sola cartilagine e di condrociti appare un po' limitativo. Lorizzonte si è allargato. La cartilagine è un tessuto non più valutato a sé stante, ma appartenente a un più complesso sistema ossocartilagine le cui interazioni non sono ancora completamente chiare. Per non parlare dell'influenza su questo sistema dell'ambiente articolare (membrana e liquido sinoviale) e dei suoi delicatissimi equilibri. Dai "condrociti svedesi" in sospensione si è passati all'uso di supporti ("scaffold") solidi, più facilmente maneggiabili e applicabili addirittura per via artroscopica, fino a una ulteriore evoluzione della ricerca che oggi sta differenziando le proprie energie in due diversi filoni: quello delle cellule (fattori di crescita, mesenchimali, terapia genica) e quello degli "scaffold" sintetici o biomimetici.

In questo fiorire di proposte di suggestioni, di idee, di spesso "facili" entusiasmi, l'ortopedico, meccanicista per formazione e mentalità si trova naturalmente un po spiazzato. Moderno apprendista stregone, rischia di perdere il controllo delle forze sconosciute che si trova a gestire, senza conoscerne esattamente la natura, la potenzialità, l'efficacia, i limiti

Scopo di questo numero monografico è proprio quello di fare il punto sullo stato dell'arte oggi. Anzitutto per puntualizzare e capire esattamente di che cosa si sta parlando e poi per circoscrivere le attuali applicazioni cliniche delle biotecnologie applicate alla chirurgia articolare del ginocchio, distinguendo il grano dal loglio: ciò che è sperimentale da ciò che può essere considerato a ragion veduta applicabile e utilizzabile con lo scopo di curare effettivamente le numerose patologie artico- lari del ginocchio, con l'aspettativa di un buon risultato.

L'Istituto Gaetano Pini, da sempre e tradizionalmente culla dell'ortopedia italiana, non poteva mancare l'occasione di ospitare con orgoglio, nell'ambito della propria rivista, le autorevoli voci di tutti quei gruppi italiani che oggi stanno dando un grande contributo, non solo a livello nazionale, ma soprattutto a livello internazionale, allo sviluppo delle biotecnologie nel trattamento della patologia articolare del ginocchio

Questa vuole essere una iniziativa scientifica seria, che ha come scopo soprattutto quello di far conoscere, di puntualizzare, di definire, di limitare. Lungi da noi fare del sensazionalismo gratuito.

Per tornare al paragone iniziale, vogliamo guardare a cosa si sta facendo nella grande vallata del "biotech" con spirito critico, mossi da un'unica stella polare: il sapere scientifico. Per questo, il lungo viaggio che avrà occasione di fare chi leggerà la rivista sarà concluso dalle considerazioni

finali, un invito alla meditazione e alla prudenza, di un grande maestro della chirurgia del ginocchio. Che il "biotech" rappresenti una grande promessa, ma anche una grande scommessa è oggi evidente. Probabil-mente fra qualche anno gran parte di quanto scritto su questa rivista risulterà superato, scavalcato, in alcuni casi definitivamente archiviato. Magari ritroveremo anche alcuni germogli fioriti dai molti semi gettati nel terreno in questi anni.

Ma è altrettanto indubbio che la strada intrapresa è di quelle da cui non si torna indietro, e che quindi anche l'approccio di chi per decenni si è abituato a ragionare in termini meccanici, riparativi e sostitutivi deve arricchirsi e allargarsi inevitabilmente al concetto di rigenerazione.

M. Berruto Struttura Semplice Interdipartimentale di Chirurgia Articolare del ginocchio, Istituto Ortopedico G. Pini, Milano 